\title{
Process Simulation of Shear Band on Initial Defect in Soil
}

\author{
SHIWEI HOU ${ }^{1, a}$, SHIJIAN HU ${ }^{1, b}$, SHAOPO GUO ${ }^{1, c}$ and XIN ZHANG ${ }^{1, d}$ \\ ${ }^{1}$ School of Civil Engineering, Shenyang Jianzhu University, Shenyang 110168, China \\ ahsw1375@126.com, b1534722335@qq.com , c1029403281@qq.com, d1002049610@qq.com
}

Keywords: shear band; initial defect; end effect; deformation process

Abstract. Formation mechanism of shear band in normal consolidated soil were analyzed based on Modified Cam-Clay model under triaxial condition. The end effect and initial defect were considered. The results proved that end restraint and initial defect can induce and control the formation process and shape of shear band. The stress and strain relationship of different part were obtained to present the bifurcation character and stress axial rotation. The interaction of different boundary and initial defect settings was analyzed in order to obtain the mechanism and deformation process of shear band.

\section{Introduction}

Progressive development of shear band is a hot spot and a difficult point on geotechnical research. The formation mechanism of shear band is the research base on both soil body stability and progressive failure. The remarkable understanding to process of shear band would have great helpful to the civil engineering design and construction.

Numerical simulation can be used as methods of finite difference, finite element, discrete element method and so on. Zienkiewicz [1] first proposed the adptive mesh technique to refresh the finite mesh in order to satisfy the failure question. Belytschko et al [2] raised extended finite element thought and meshless method. Wang et al [3] used discrete element to research particle breakage under high pressure and plane strain conditions. Powrie et al [4] researched end friction of triaxial test by using particle flow method. Castelli et al [5] simulated the propagation law of shear band in defect material using boundary element method. $\mathrm{Lu} \mathrm{[6]} \mathrm{came} \mathrm{forward} \mathrm{principal} \mathrm{stress} \mathrm{conditions} \mathrm{of} \mathrm{shear} \mathrm{band} \mathrm{process}$ and failure under plane strain conditions.

Formation mechanism and development process of shear band under triaxial condition using modify cam model was analyzed in this paper. The results show that elements in non shear band area has the bifurcation character and stress axial rotation character.

\section{Formation Process of Shear Band}

The formation process of shear band is complicate and effected by boundary conditions. For the same initial defect and different boundary displacement condition, the formation processes of shear band are completely different.

The soil parameters used in constitutive model was the same as parameters of Sam [7], in which $\alpha=50 \mathrm{kPa}, \beta=1.0, \mathrm{M}=1, \lambda=0.174, \kappa=0.026, \mathrm{e}_{0}=0.889, \mu=0.28, \mathrm{k}=25 \mathrm{~mm} / \mathrm{s}, \xi=1.0$. Pore pressure element CPE8RP was used. Sample was cylindrical specimen, size was $50 \mathrm{~mm} \times 100 \mathrm{~mm}$. The simulation process was two steps, the cell pressure $100 \mathrm{kPa}$ was put in first step, strain controlled loading under unconstrained condition was put in second step. Top and bottom of sample were displacement boundary conditions, left and right boundaries were stress conditions. The loading method was strain control.

Condition 1 . The sample with no end effect and initial defect is simulated firstly, in order to compare with random initial defect conditions. The Fig. 1 is the vertical displacement and vertical log strain results under $20 \mathrm{~mm}$ vertical compression. Based on the simulation results, we can see that the vertical displacement is increased with height, and the vertical strain is uniform. 
The formation mechanism of sample is analyzed using stress-time results of representative elements. The representative element 1633 is located at middle of sample surface. The representative element 1644 is located at the upper end of sample surface. As fig. 2 shows that the stress of 1,2,3 are all equal for the two elements under no end effect and initial defect condition.

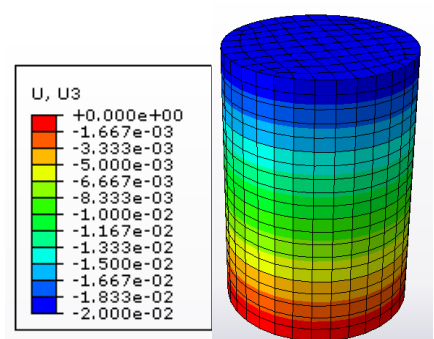

(a) vertical displacement

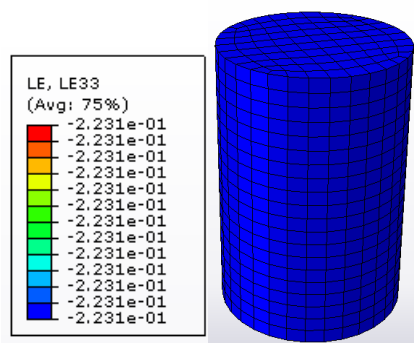

(b) vertical log strain

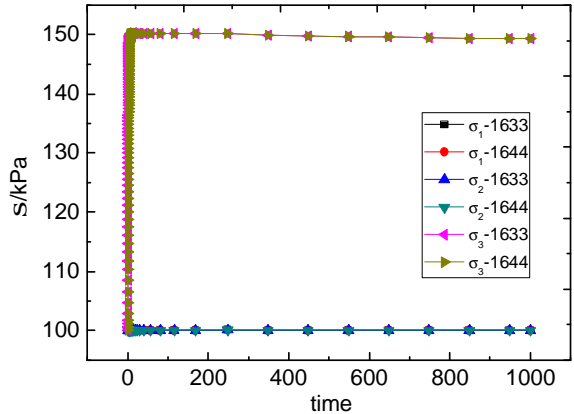

Fig.2 Stress of representative elements Fig.1 Vertical displacement and log strain of sample

Condition 2. End effect is a kind of boundary condition during the triaxial text cause by texting equipment. It can't be avoid, but has the important effect in sample text result.Now considering the end effect of triaxial testing, the following simulation is the result with upper end effect. The fig. 3 is the vertical displacement and vertical log strain results under $20 \mathrm{~mm}$ vertical compression. Based on the simulation results, we can see that the vertical displacement is increased with height, but the vertical strain is not uniform. The whole deformation is constrained by upper end.

The representative elements 1633 and 1644 are analized in order to compare with different boundary conditions. The stress and strain curves with calculation time are showed in fig.4. The representative element 1633 is located at middle of sample surface, so the strains of different directions are all larger than the results of element 1644 which located at end.

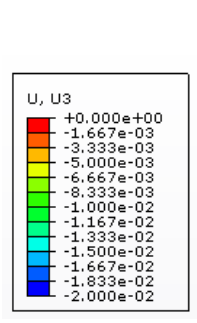

(a) vertical displacement

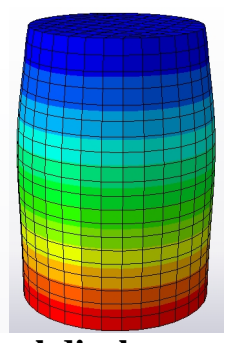

Fig.3 Vertical displacement and log strain of sample

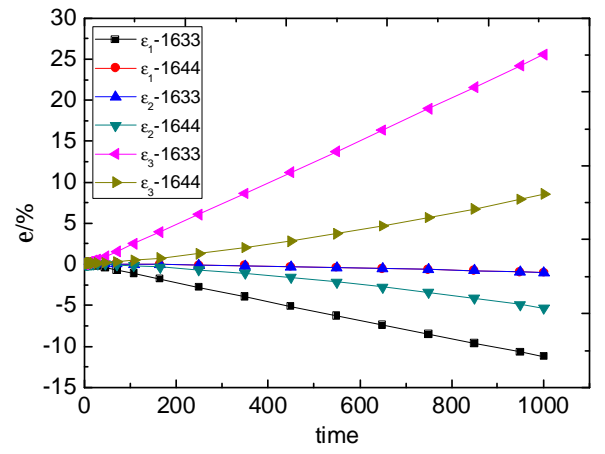

(a) strain-time relationship

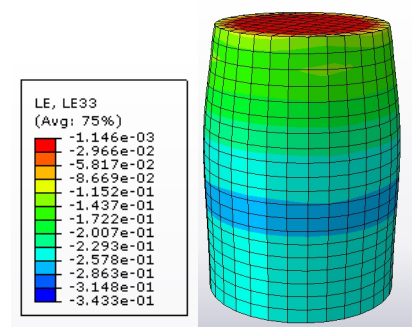

(b) vertical log strain

Fig.4 Strain and stress of representative elements

Compared the end effect condition with no end effect condition. We can see that the horizontal and vertical stress of element 1633 which loacted at middle surface of sample is decresed under end effect action. But the stress results of element 1644 are all increased when compared with no end effect. 
Condition 3. Initial defect is also a kind of boundary condition during the triaxial text. Different with end effect, it's from the sample internal part because of the material inhomogeneity. The following calculation is simulating the initial defect that cause by sample installation or internal inhomogeneity. The upper end element 1644 was chosen as initial defect element. The normal element parameters are as follows, $\alpha=50 \mathrm{kPa}, \beta=1.0, \mathrm{M}=1.4, \lambda=0.174, \kappa=0.026, \mathrm{e}_{0}=0.889, \mu=0.28, \mathrm{k}=25 \mathrm{~mm} / \mathrm{s}, \xi=1.0$, while initial yeild strength $\alpha=15 \mathrm{kPa}, M=1$ for initial defect element. Other 5 representative elements were chosen to be researched. These elements and vertical strain were shown in fig.5(a),(b), other nephogram results are shown in fig.5(c),(d).

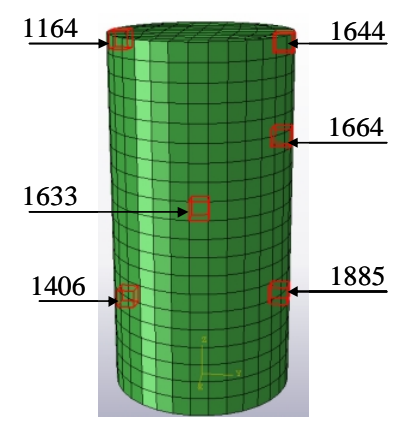

(a) representative elements

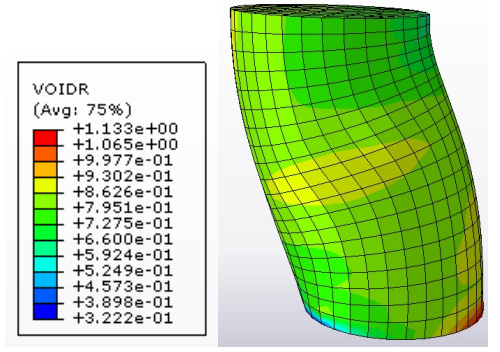

(c) Void ratio nephogra

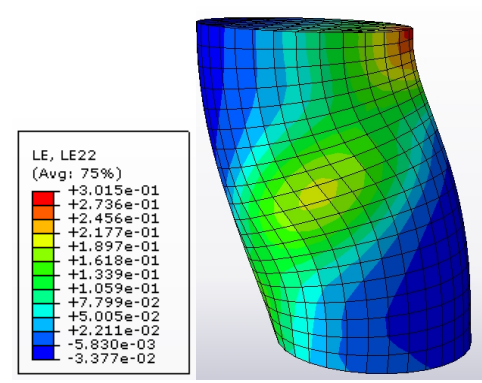

(b) Strain nephogram

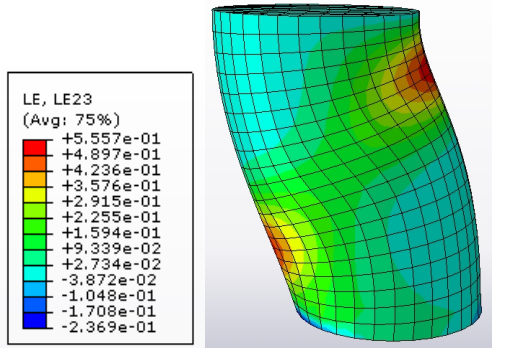

(d) Log shear strain nephogram

Fig.5 Boundary conditions and results

Form the nephogram results, the whole sample inclination was caused by one initial defect element, strain localziation area formed under undrained condition. Representative elements $1164,1644,1664,1633,1406,1885$ are used to analyze the process of shear band. The elements 1664,1633,1406 are in the main shear band. The elements 1164 and 1885 are located at top and bottom no shear band area respectively. Fig. 6 is the stress and strain curve with time of the initial defect element. It can be seen that stress and strain of 1644 are increasing with calculation time, but stress present the two steps character. The first step is stress of three directions increasing rapidly to inflection point. The second step is all three stresses increasing slowly to the peak point.

Fig. 7 is the stress and strain curve with time of the non shear band area elements. In fig.7(a) the vertical stress is rapidly decreasing to horizontal stress after peak value, then the max main stress turn into min main stress which means that the main stress axis has changed. The strain present the compression to extension character. The conclusion is match with the strain results and also shows the formation process of shear band. 


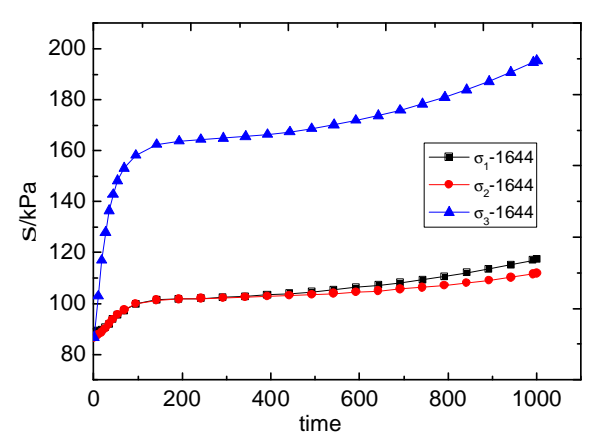

(a) stress-time relationship

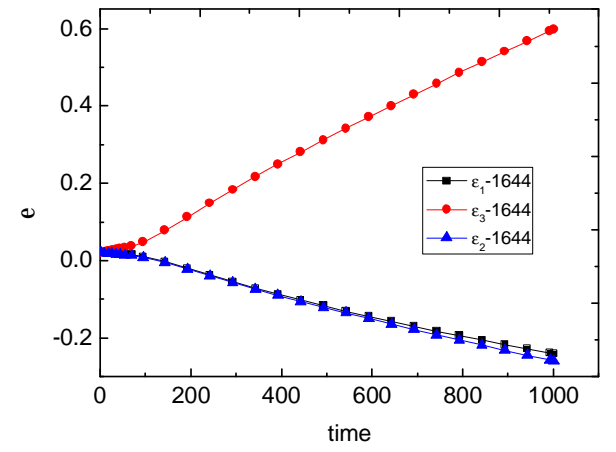

(b) strain-time relationship

Fig.6 Stress and strain curves of initial defect element

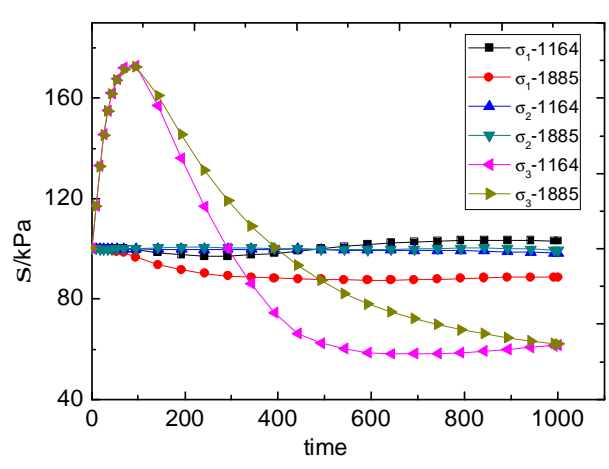

(a) stress-time relationship

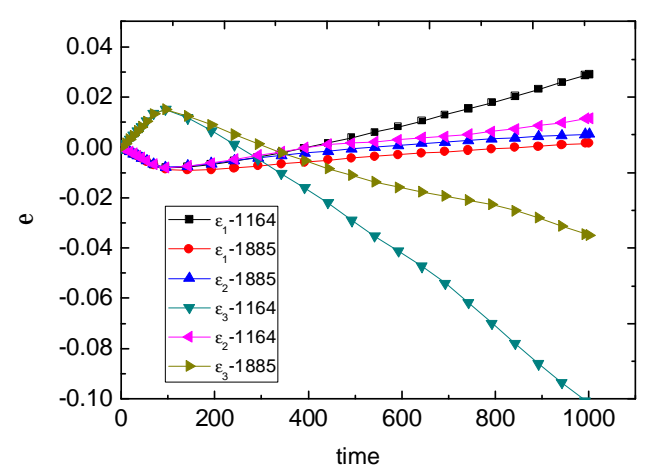

(b) strain-time relationship

Fig.7Stress and strain curves in non strain location area

Fig. 8 is the stress and strain curve with time in shear band area elements. In fig.8(a) the vertical stress is slowly decreasing after peak value, and the other horizontal stress present the bifurcation behavior after the peak value. One horizontal stress is nearly equal, and the other decrease slowly. This element is located at the middle surface of the sample. Elements 1406 and 1664 are both located at strain localization area, but their max main stresses are more likely to character of initial defect element 1644 , the other stresses are both increasing slowly during the loading process, and has no significant bifurcation character.

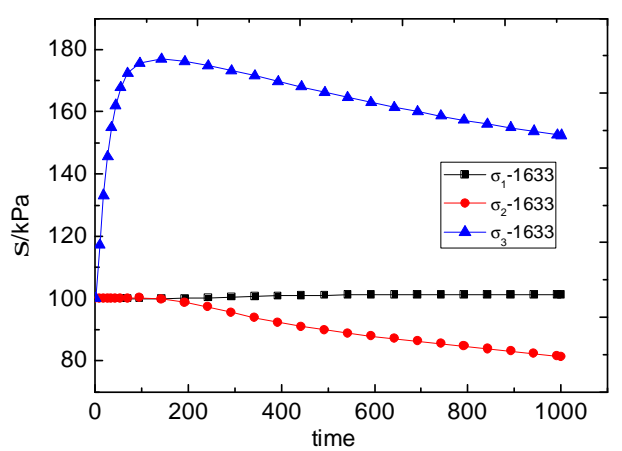

(a) stress of element 1633

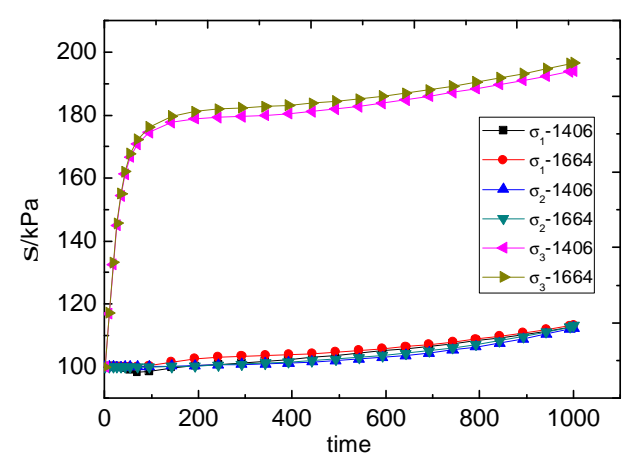

(b) stress of element 1406 and 1664

Fig.8 Stress and strain curves in strain location area

Now take the $1406,1633,1885$ representative elements as example to analysis the formation mechanism of shear band under initial defect condition. The fig. 9 shows the stress and time relationship at different location of sample. The whole stress can be divided into two main phases. The first phase is consistent deformation for different location elements. The time for this phase is short, the whole sample happen elastic deformation. After the vertical stress peak value the vertical stress present the obvious bifurcation character, the stress has increasing slowly, decreasing slowly and decreasing rapidly three conditions. For reflection area element, the max main stress turn into min main stress which means that the main stress axis has changed. The conclusion is match with the strain results and also shows the formation process of shear band. 


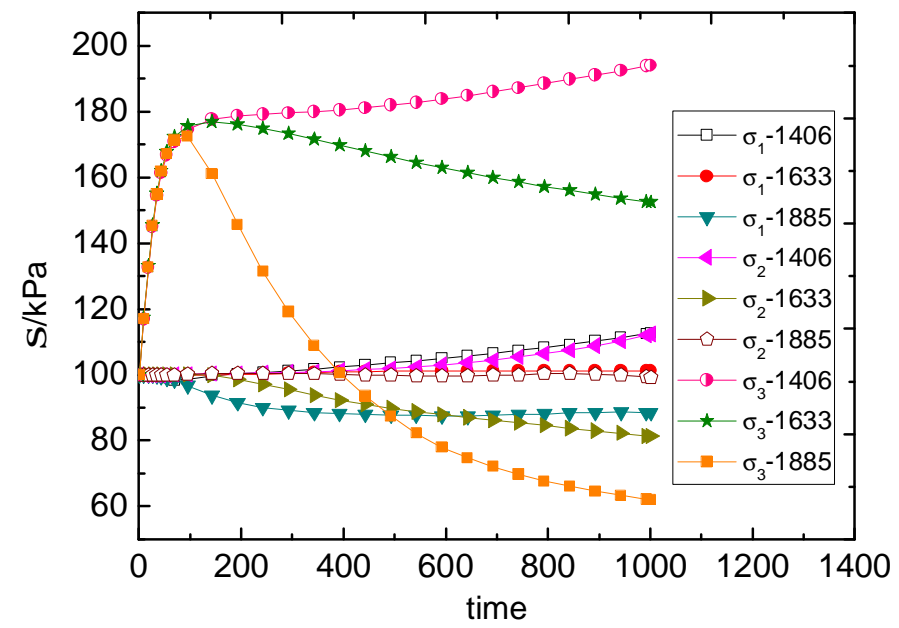

Fig.9 Stress of representative elements

Now put the end effect, initial defect and uniform conditions together as fig.10 shows. Take the same representative elements as example to analysis the formation mechanism of shear band. The stress peak value is different for 3 conditions. The initial defect element present the obvious difference in zero point and peak point. The element 1644 has lower consolidation stress because of the lower initial yeild strength. The pore pressure with time of 3 conditions are presented in fig.11. the pore pressure of initial defect condition has significant non-linear character compared with the other two conditions .

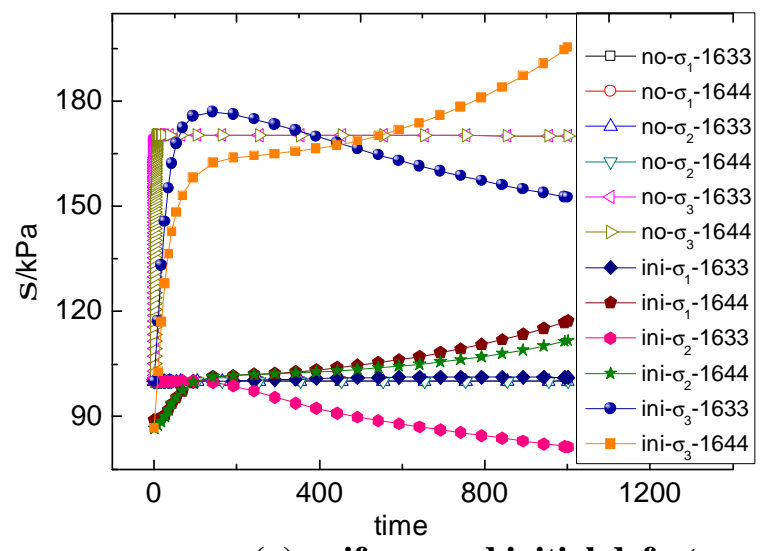

(a) uniform and initial defect conditions

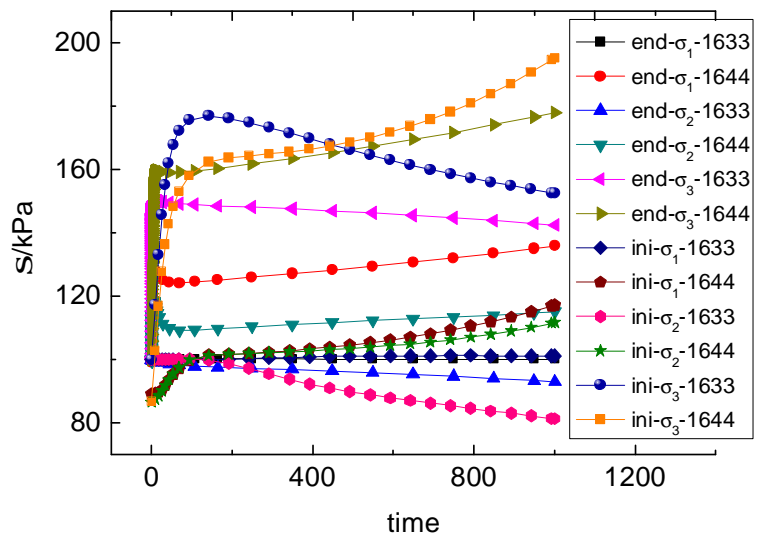

(b) end effect and initial defect conditions

Fig.10 Stress curves for different calculation conditions

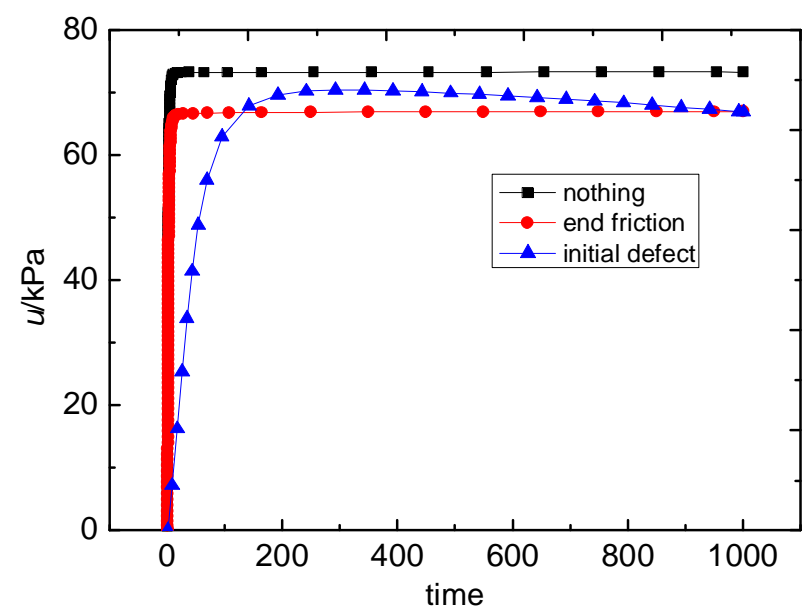

Fig.11 Comparison of pore pressure 


\section{Conclusions}

Formation mechanism and development process of shear band under triaxial condition were researched using numerical simulation method.

The results proved that end restraint and initial defect can induce and control the formation process and shape of shear band in triaxial texting. The interaction of different boundary and initial defect settings can decide deformation process of shear band. End effect has effect on the top and bottom stress, can't cause the whole incline of the specimen. But one initial defect can cause the incline of the specimen. It means that the shear band formation has relationship with boundary symmetry.

The unsymmetrical initial defect induced the shear band, during the deformation process, representative elements of different location present the bifurcation character and stress axial rotation character. The max main stress can turn into min main stress. The strain present the compression to extension character. The conclusion is match with the plane strain results and also shows the formation process of shear band.

\section{Acknowledgements}

This work was financially supported by the National Natural Science Foundation of China (51308355), Project supported LiaoNing Province, colleges and universities outstanding young scholar growth plan (LJQ2014058), Ministry of Housing and Urban-Rural Development of the People's Republic of China Project (2015-K3-025)and Shenyang Jianzhu University Subject Construction Project(XKHY-09).

\section{References}

[1] O C Zienkiewicz, M S Huang, M Pastor. Int. J. Numer. Anal. Meth Geomech, Vol. 19(1995), p. 127

[2] T Belytschko, T Black. Int. J. Numer. Meth Engng, Vol. 45(1999), p.601

[3] J F Wang, H B Yan. Procedia Engineering, Vol. 14(2011), p.1713

[4] W Powrie, Q Ni, R M Harkness, et al. Geotechnique, Vol. 55(2005), p. 297

[5] M Castelli, A Allodi, C Scavia. Int. J. Numer. Anal. Meth Geomech, Vol. 33(2009), p.1561

[6] D C Lu, Y P Yao, A N Zhou. Chinese Journal of Rock Mechanics and Engineering, Vol. 25(2006), p.2320, in Chinese

[7] H Sam. Applied soil mechanics with ABAQUS applications. (John Wiley \& Sons, Inc, USA 2007)

[8] X L Du, S W Hou, D C Lu, et al. Rock and Soil Mechanics, Vol. 33(2012), p.1281, in Chinese 